\title{
Paciente con síndrome metabólico
}

\author{
MARÍA MORENO COLLADO \\ al316134@uji.es \\ Águeda Cervera Gasch \\ cerveraa@uji.es
}

\section{Resumen}

Introducción: El síndrome metabólico es el conjunto de patologías que generan riesgo de desarrollar una enfermedad cardiaca o diabetes mellitus tipo 2. El objetivo de la intervención es mejorar la calidad de vida potenciando las medidas higiénico-dietéticas y realizar intervenciones para paliar las complicaciones derivadas por las diferentes patologías metabólicas. Metodología: Se desarrolla un caso clínico basado en una paciente de 81 años que sufre diabetes mellitus tipo 2 y enfermedad cardiovascular debido a un síndrome metabólico, caracterizado por hipertensión arterial, glucemia elevada en sangre, niveles sanguíneos de triglicéridos elevados, bajos niveles de HDL y exceso de grasa central. Se realizan diferentes escalas de enfermería y pruebas analíticas para valorar el estado de la paciente tanto físico como psíquico. Se realiza la valoración con las escalas de Morisky-Green Levine, Pfeiffer, Barber, Barthel y APGAR familiar, también se solicita un análisis y toma de constantes y glucemia de tres sesiones. Resultados: Se crea un plan de intervención modificando la dieta y el ejercicio físico basado en una búsqueda de la mejor evidencia disponible en la base de datos PubMed. Discusión: Nuestras acciones van dirigidas a que la paciente mejore su estado de salud intentando conseguir una disminución de la glucemia y una disminución de peso y perímetro abdominal para evitar estar en sobrepeso y la posibilidad de complicar su enfermedad cardiovascular. Estas intervenciones pueden mejorarse modificando su dieta y su actividad física diaria.

Palabras clave: síndrome metabólico, cuidados de enfermería, prevención, dieta, ejercicio físico.

\section{Abstract}

Introduction: Metabolic syndrome is a set of conditions that creates a risk of developing heart disease or diabetes mellitus type II. The aim was to improve quality of life by enhancing hygiene and diet measures and interventions to alleviate any complications arising from different metabolic diseases. Methodology: A case was developed based on an 81-year-old patient with diabetes mellitus type II and cardiovascular disease due to metabolic syndrome characterised by high blood pressure, high blood glucose, blood levels of triglycerides, low HDL levels and excess abdominal fat. Different nursing scales and laboratory tests were performed to assess the patient's physical and mental state. 
An evaluation was made with the Morisky-Green Levine, Pfeiffer, Barber, Barthel and family APGAR scales. A blood test was requested, vital signs and were checked blood glucose during three sessions. Results: An intervention plan was devised by modifying diet and exercise based on a search for the best evidence available in the PubMed database. Discussion: Our actions aimed to improve the patient's health by attempting to lower blood glucose, lose weight and reduce waist circumference to avoid being overweight and the possibility of complicating cardiovascular disease. These interventions can be improved by modifying diet and daily physical activity.

Keywords: Metabolic syndrome, Nursing Care, Prevention, Diet, Physical exercise.

\section{Introducción}

El síndrome metabólico, también denominado síndrome $X$ o síndrome de resistencia a la insulina, es un grupo de factores que generan riesgo de desarrollar una enfermedad cardiaca y diabetes tipo 2. Los factores de riesgo más importantes son la obesidad central y la resistencia a la insulina (Gucev, Tasic y Polenakovic, 2015). La insulina es una hormona secretada por el páncreas cuya función es el control de azúcar en sangre. La resistencia a la insulina significa que algunas células no utilizan eficazmente la insulina y esto induce un aumento de azúcar en sangre, por lo que provoca un incremento de grasa corporal (Gupta, Mishra, Kumar, y Mishra, 2015).

Otros factores que influyen son la edad avanzada, los genes, los cambios en las hormonas masculinas y femeninas del estrés y la falta de ejercicio.

Con la presencia de tres o más de los factores siguientes en la persona, se considera que sufre síndrome metabólico (Korotaeva, Novikoya y Loginova, 2016):

- Hipertensión arterial $\geq 130 / 85 \mathrm{mmHg}$.

- Glucosa en la sangre $\geq 100 \mathrm{mg} / \mathrm{dL}$.

- Niveles sanguíneos elevados de triglicéridos $\geq 150 \mathrm{mg} / \mathrm{dL}$.

- Bajos niveles sanguíneos de HDL ( $\leq 40 \mathrm{mg} / \mathrm{dL}$ en hombres y $\leq 50 \mathrm{mg} / \mathrm{dL}$ ).

- Perímetro abdominal elevado ( $>102 \mathrm{~cm}$ en hombres y $>88 \mathrm{~cm}$ en mujeres).

Debido a la importancia del síndrome metabólico y del problema de salud que supone, merece especial importancia el crear estrategias de prevención de estas patologías y, en el caso de que ya estén establecidas, es de vital importancia evitar las complicaciones que se puedan derivar. Por tanto, se plantean los siguientes objetivos para este estudio:

Objetivo general: mejorar la calidad de vida potenciando las medidas higiénico-dietéticas y realizar intervenciones para paliar las complicaciones derivadas por las diferentes patologías metabólicas.

Objetivos secundarios: recogida de datos sobre las enfermedades y sus posibles complicaciones; realizar intervenciones adecuadas y consensuadas con la paciente para mejorar sus hábitos dietéticos, de ejercicio y sobre el tratamiento pautado. 


\section{Método}

Se realizó el caso clínico que se presenta sobre una paciente diagnosticada de síndrome metabólico. Mujer de 81 años con diabetes mellitus tipo 2, hipertensa, sobrepeso grado 2 y con tratamiento de anticoagulantes. Patologías que caracterizan el síndrome metabólico. Además, relacionado con la edad y una red de apoyo familiar deficiente, es una paciente de riesgo debido a la posibilidad de no seguir el tratamiento. Es una usuaria que consta, desde el 2001 hasta el 2015, con un registro de las vacunas administradas: se ha vacunado anualmente de la gripe, lleva las tres dosis de vacuna antitetánica y, además, está inmunizada contra el pneumococo y la gripe aviar H1N1. El tratamiento farmacológico que sigue consta de Sintrom según la pauta, Alopurinol $300 \mathrm{mg} 1 \mathrm{comprimido/día,} \mathrm{Lantus} 38 \mathrm{UI}$ al desayuno, Espironolactona $25 \mathrm{mg} 1$ comprimido/día, Furosemida $40 \mathrm{mg} 1 \mathrm{comprimido/día,} \mathrm{Pravastatina} 40 \mathrm{mg} 1 \mathrm{comprimido/día,}$ Irbesartan/Hidroclorotiazida 300/25 1 comprimido/día, Bisoprolol $10 \mathrm{mg} 1 \mathrm{comprimido/día} \mathrm{y}$ Pantoprazol $40 \mathrm{mg} 1 \mathrm{comprimido/día} \mathrm{(Andugar-Hernández} \mathrm{J.} \mathrm{y} \mathrm{cols.,} \mathrm{2016).}$

La paciente tiene un peso de $70,5 \mathrm{~kg}, 154 \mathrm{~cm}$ de talla y un índice de masa corporal de $29,7 \mathrm{~kg} / \mathrm{m}^{2}$. Además, valoramos las constantes vitales de tres consultas y el último análisis (vénase las tablas 1 y 2 ).

Tabla 1

Constantes vitales

\begin{tabular}{cccc}
\hline & tas/tad & fc & Glucemia \\
\hline 1 & $139 / 74 \mathrm{~mm} \mathrm{Hg}$ & 76 & $103 \mathrm{mg} / \mathrm{dl}$ \\
2 & $119 / 68 \mathrm{~mm} \mathrm{Hg}$ & 79 & $143 \mathrm{mg} / \mathrm{dl}$ \\
3 & $127 / 77 \mathrm{~mm} \mathrm{Hg}$ & 68 & $147 \mathrm{mg} / \mathrm{dl}$ \\
\hline
\end{tabular}

Tabla 2

Última analítica

\begin{tabular}{lccc}
\hline & ANÁLISIS CLíNICOS & \\
\hline $\begin{array}{l}\text { Bioquímica Sueros Analclin } \\
\text { Urea }\end{array}$ & 69 & ${ }^{*} \mathrm{mg} 7 \mathrm{dl}$ & $(10.0-50.0)$ \\
Creatinina & 1.58 & ${ }^{*} \mathrm{mg} / \mathrm{dl}$ & $(0.50-1.20)$ \\
$\begin{array}{l}\text { Filtrado Glom. Estimado } \\
\text { CKD-EPI }\end{array}$ & 31.80 & ${ }^{*} \mathrm{ml} / \mathrm{min} / \mathrm{sup}$ & $(60.0-200.0)$ \\
Sodio & 140 & & \\
Potasio & 5.20 & $\mathrm{mmol} / \mathrm{l}$ & $(134-145)$ \\
Cloro & 100 & ${ }^{*} \mathrm{mmol} / \mathrm{l}$ & $(3.50-5.00)$ \\
Úrico & 6.2 & $\mathrm{mmol} / /$ & $(95-105)$ \\
Hormonas Analclin & & $\mathrm{mg} / \mathrm{dl}$ & $(2.4-7.0)$ \\
Glicohemoglobina A1C $(\%)$ & 7.8 & & \\
\hline
\end{tabular}


Se observó una tensión arterial normal gracias a la toma de hipotensores para controlar la posible subida que pudiera conllevar riesgos para su enfermedad cardiovascular, lo que también podría surgir debido a su sobrepeso y obesidad central. Sufrió con anterioridad un accidente cerebrovascular (ACV), le implantaron un DAI-TRC (desfibrilador automático implantable - tratamiento de resincronización cardiaca), lo que le supuso la toma de forma crónica de acenocumarol (Sintrom).

La glucemia varía dependiendo de la ingesta de comida de la paciente ya que no sigue una dieta adecuada para su estado de salud. El intervalo adecuado es $70-130 \mathrm{mg} / \mathrm{dl}$, pero la paciente en las últimas consultas lo supera $(143,147)$. También tiene elevado el porcentaje de $\mathrm{HbA}_{1} \mathrm{C}$ que debería ser inferior a $7 \%$ y está en $7,8 \%$. Se ve un deterioro de la filtración glomerular, $31,80 \mathrm{ml} / \mathrm{min} / \mathrm{sup}$, siendo $60,0-200,0$ el intervalo correcto, además del aumento de urea y creatinina (Di Micco y cols., 2013)

Se realizan diferentes test con los siguientes resultados:

- Test de Morisky-Green Levine: buen cumplimiento del tratamiento.

- Test de Pfeiffer: puntuación normal.

- Test de Barber: situación de riesgo.

- Test de Barthel: independiente.

- Test APGAR familiar: disfuncional grave.

Se le insiste en la ingestión de las cantidades adecuadas de alimentos además de que sea una dieta hiposódica, baja en grasas y sin azúcares; también se le aconseja ejercicio diario como puede ser ir caminando media hora todos los días sin cansarse y que continúe asistiendo a sus controles mensuales.

Los diagnósticos de enfermería son:

- Desequilibrio nutricional por exceso.

- Manejo ineficaz del régimen terapéutico.

- Déficit de autocuidado: baño/cuidado personal.

- Riesgo de soledad.

- Tendencia a adoptar conductas de riesgo para la salud.

- Conductas generadoras de salud (diabetes/HTA).

La intervención enfermera se basa en la creación de un plan de intervención para modificar los hábitos dietéticos y de ejercicio físico. Para realizar esta intervención enfermera, se realizó una búsqueda bibliográfica en la base de datos PubMed, con la finalidad de aplicar los cuidados de enfermería bajo la mejor evidencia científica disponible. Los descriptores utilizados fueron metabolic sindrome $X$, exercise y diet. Se establecieron los siguientes criterios de inclusión:

- Artículos escritos durante los últimos 5 años.

- Artículos escritos en castellano o inglés.

- Artículos recuperables a texto completo.

- Estudios realizados en humanos.

Tras la lectura de título y abstract, se incluyeron en la revisión los artículos relacionados con el objetivo del estudio.

Como resultados se obtuvieron $n=82 ; 15,9 \%$ aplicables al tema tratado y $84,14 \%$ descartados. 


\section{Resultados}

Tras la búsqueda realizada en la base de datos PubMed, se obtuvieron los siguientes resultados: un total de 82 artículos fueron recuperados. El 84,14\% $(n=69)$ se descartaron por no estar relacionados con el tema de estudio. El 15,9\% $(n=13)$ fueron utilizados para planificar la intervención enfermera.

La primera intervención realizada fue la pauta de una dieta con las siguientes características: una dieta hipocalórica, hiposódica para disminuir la tensión arterial, diabética disminuyendo la ingesta de azúcares y baja en vitamina $\mathrm{K}$ para mantener el INR en valores adecuados debido a las alteraciones de la paciente. Ya que la revisión de la literatura indica que la reducción de peso corporal disminuyendo la ingesta alimentaria aproximadamente unas $500 \mathrm{kcal} /$ diarias es el primer factor eficaz demostrado en la disminución del síndrome metabólico, se aconsejará una dieta equilibrada siendo ejemplar la dieta mediterránea. La siguiente intervención realizada está relacionada con la realización de ejercicio físico diario. Diferentes estudios (como, por ejemplo, Ruzafa-Martinez, Lopez-Iborra, Moreno-Casbas y Madrigal-Torres, 2013; Thaiss, Zmora, Levy y Elinav, 2016)

indican que los mejores beneficios se obtienen caminando durante sesenta minutos sin agotarse, aunque en este caso, debido al DAI-TRC, serían treinta minutos. Como última intervención se recomienda continuar asistiendo a las revisiones de glucemia mensuales aparte del control diario en el domicilio. También se controlará la tensión mensualmente, a no ser que aparezcan valores alterados, en cuyo caso se modificarán las fechas de consulta hasta su estabilización. Del mismo modo, se considera imprescindible controlar los valores del INR de forma mensual si no hay alteraciones.

Como pauta de mantenimiento, se considera necesario la realización de un análisis cada medio año para valorar la hemoglobina glicosilada y de una revisión de pies, electrocardiograma y fondo de ojo de forma anual, por lo que se derivará a la asistencia especializada.

\section{Discusión y conclusión}

Las acciones realizadas van dirigidas a que la paciente mejore su estado de salud intentando conseguir una disminución de la glucemia al comer menos azúcares y conseguir una disminución de peso y de perímetro abdominal para evitar estar en sobrepeso y evitar también la posibilidad de complicar su enfermedad cardiovascular al modificar su dieta y su ejercicio diario (Ageno y cols., 2015; Tzoran y cols., 2014).

Por el contrario, no podemos evaluar la efectividad de estas intervenciones debido a que en la última consulta de hipertensión y Sintrom presentaba un tono de piel pálido y lentitud al hablar. La literatura indica que no hay diferencia significativa entre una dieta de proteínas vegetales o de animales, por lo que una combinación de ambas es ideal (Barrio Gómez de Agüero, García-Hernández y Gartner, 2009; Smirnova, Barilo y Smolnikova, 2016). La ingesta de lácteos también favorece la disminución de esta alteración, aunque las grasas saturadas de otros alimentos estén contraindicadas (Di Micco y cols., 2013). Se ha demostrado que la actividad física favorece la captación de glucosa, por lo que los receptores de insulina mejoran, disminuye el perímetro abdominal al mismo tiempo que también disminuye la tensión arterial (Nalado y cols., 2015). Durante la anamnesis comentó que comía poco, que había vomitado sin sangrado y que hizo una deposición blanda.

Durante la exploración, en la toma de tensión salieron unos valores un poco bajos, TAD:100, TAS:60 y FC: normal. El valor de INR estaba dentro de la normalidad, 2.2, y la glucemia alta. Se avisa al médico para que lo valore, quien solicita un electrocardiograma con resultado 
normal. A la palpación se queja de dolor intenso en el epigastrio. Durante la conversación enfatizamos en las deposiciones en las que se concluye que eran melénicas y se prosigue a una derivación a urgencias hospitalarias. El resultado de urgencias fue una hemorragia digestiva alta.

Por último se seguirá valorando su estado e insistiendo en los cambios de hábitos una vez esté dada de alta en el hospital y sea remitida para el seguimiento en atención primaria.

\section{Referencias bibliográficas}

Andugar Hernández, J., Fortun-Sánchez, M., Arias-Rodríguez, I., Gómez-Rodríguez, I., Arroyo-Revenga, M. P., Guerrero-Llamas, L. y cols. (2016). Guías de intervención en hipertensión y riesgo cardiovascular para enfermería. Madrid: Imprenta Tomás Hermanos.

Ageno, W., Samperiz, A., Caballero, R., Dentali, F., Di Micco, P., Prandoni, P., Becattini, C., Uresandi, F., Verhamme, P. y Monreal, M. (2015). Duration of anticoagulation after venous thromboembolism in real world clinical practice. Thrombosis Research, 135, 666672.

Barrio Gómez de Agüero, M. I., García Hernández, G. y Gartner, S. (2009). Protocolo de diagnóstico y seguimiento de los pacientes con fibrosis quística. Anales de Pediatría, 71, 250-264.

Di Micco, P., Ruiz-Giménez, N., Nieto, J. A., Aujesky, D., del Molino, F., Valle, R., Barrón, M., Maestre, A. y Monreal, M. (2013). Platelet count and outcome in patients with acute venous thromboembolism. Thrombosis and Haemostasis, 110, 1025-1034.

Gucev, Z., Tasic, V. y Polenakovic, M. (2015). 4th Rare Disease South Eastern Europe (SEE) Meeting Skopje, Macedonia (14 de noviembre de 2015). Prilozi (Makedonska Akademija Na Naukite I Umetnostite. Oddelenie Za Medicinski Nauki), 36, 151-156.

Gupta, V., Mishra, S., Kumar, S. y Mishra, S. (2015). Association of Circulating Orexin-A Level With Metabolic Risk Factors in North Indian Pre Menopausal Women. Indian Journal of Physiology and Pharmacology, 59, 422-427.

Korotaeva, T. V., Novikoya, D. S. y Loginova, E. Y. (2016). Cardiovascular risk in patients with psoriatic arthritis. Terapevticheskil Arkhiv, 88, 102-106.

Nalado, A. M., Musa, B. M., Gezawa, I. D., Muhammad, H., Ibrahim, D. A. y Uloko, A. E. (2015). Prevalence of metabolic syndrome among apparently healthy adults in a rural community, in north-western Nigeria. Nigerian Journal of Medicine: Journal of the National Association of Resident Doctors of Nigeria, 24, 323-330.

Ruzafa-Martinez, M., Lopez-Iborra, L., Moreno-Casbas, T. y Madrigal-Torres, M. (2013). Development and validation of the competence in evidence based practice questionnaire (EBP$\mathrm{COQ}$ ) among nursing students. BMC Medical Education, 13, 19.

Smirnova, S. V., Barilo, A. A. y Smolnikova, M. V. (2016). Hepatobiliary System Diseases as the Predictors of Psoriasis Progression. Vestnik RossiřskoI Akademii Meditsinskikh Nauk / Rossiľskaia Akademiia Meditsinskikh Nauk, 2, 102-108.

Thaiss, C. A., Zmora, N., Levy, M. y Elinav, E. (2016). The microbiome and innate immunity. Nature, 535, 65-74.

Tzoran, I., Brenner, B., Sakharov, G., Trujillo-Santos, J., Lorenzo, A., Madridano, O., LópezSáez, J. B. y Monreal, M. (2014). Clinical outcome in patients with venous thromboembolism receiving concomitant anticoagulant and antiplatelet therapy. European Journal of Internal Medicine, 25, 821-825. 